\title{
ON THE ANGLE FOR STATIONARY RANDOM FIELDS
}

\author{
A.G. Miamee and H. Niemi \\ Hampton University, Department of Mathematics, Hampton, VA 23668, U.S.A. \\ University of Helsinki, Department of Statistics, Aleksanterinkatu 7, SF-00100 Helsinki, Finland
}

\begin{abstract}
The angle between past and future for stationary random fields on the lattice points of the plane is defined and it is shown that in contrast with other problems related to the past of random fields the positivity of the angle betweeen past and future is independent of different pasts which have been considered in the literature. It is shown that the positivity of the angle for random fields and processes is equivalent with that forming a Schauder basis. Besides this some analytic characterizations are also provided.
\end{abstract}

1. Introduction. A problem which has been proved to be useful in the prediction theory of stationary stochastic processes is the idea of the angle between past and future. Several authors have worked on this area and revealed its connection with the prediction theory of stationary stochastic processes, c.f. Helson and Szegö [4], Hunt, Muckenhoupt and Wheeden [5], Pousson [11], Sarason [13], Pourahmadi [10], and Miamee [8].

In this paper we introduce the definition of the angle for a stationary random field and prove that the crucial properties of the angle in the case of stationary processes have natural extensions to the case of stationary fields. In contrast to other problems concerning the past of random fields, we show that the positivity of the angle between past and future does not depend on the choice of different kind of pasts considered by different authors in the literature.

After setting up the necessary notation in Section 2 we prove our analytic and geometric characterization for positivity of the angle in Section 3.

The present paper is essentially based on the report [9]. In fact we reproduce some of the main results obtained already in [9]. Related results, based on [9], have been obtained also by Makagon and Salehi [7].

2. Preliminaries. In this section we introduce the notation and terminology needed in the rest of the paper. Let $X_{m n},(m, n) \in Z^{2}$, be a double sequence of random variables on a probability space $(\Omega, \mathscr{B}, \mathbf{P})$ such that $\mathbf{E} X_{m n}=0$ and $\mathbf{E}\left|X_{m n}\right|^{2}<\infty$, for all $(m, n) \in Z^{2}$. The double sequence $X_{m n},(m, n) \in Z^{2}$, is called a stationary random field if $\mathbf{E} X_{m n} \bar{X}_{r s}$ depends only on the differences

1991 Mathematics Subject Classification: Primary: 60G60.

Research was supported by AFOSR \#F 4962082 C 0009.

doi:10.5186/aasfm.1992.1712 
$m-r$ and $n-s$; i.e. $\mathbf{E} X_{m n} \bar{X}_{r s}=\varrho(m-r, n-s)$. In this case the covariance function $\varrho(m, n)=\mathbf{E} X_{m n} \bar{X}_{r s}$ is a positive definite function on the group $Z^{2}$ of lattice points of plane. It is known (cf. for example Salehi and Scheidt [13]) that there exists a non-negative measure $\mu$, defined on the Borel sets of the torus

$$
T=\{\alpha: 0 \leq \alpha \leq 2 \pi\} \times\{\beta: 0 \leq \beta \leq 2 \pi\}
$$

such that

$$
\varrho(m, n)=\int e^{-i(m \alpha+n \beta)} d \mu, \quad \text { for all }(m, n) \in Z^{2} .
$$

The measure $\mu$ is called the spectral measure of the stationary random field $X_{m n}$. If $\mu$ is absolutely continuous with respect to the normalized Lebesgue measure

$$
d \sigma=\frac{d \alpha d \beta}{4 \pi^{2}}
$$

its Radon-Nikodym derivative $w$ is called the spectral density of the field.

By $L_{\mu}^{2}$ we denote the Hilbert space of all functions on the torus which are square summable with respect to the measure $\mu$. From (2.1) it is clear that the operator

$$
X_{m n} \rightarrow e^{-i(m \alpha+n \beta)}
$$

extends to an isomorphism from $H_{X}=$ the closed linear subspace generated by all $X_{m n}$ 's, onto $L_{\mu}^{2}$. This is called the Kolmogorov isomorphism between the time domain and spectral domain.

For any subset $M$ of $Z^{2}$ we define $H_{X}(M)$ to be the closed linear subspace of $L^{2}(\Omega, \mathscr{B}, \mathbf{P})=H$, spanned by all $X_{m n}$ 's with $(m, n) \in M$. The vertical pastpresent $P_{X}^{v}$ and the vertical future $F_{X}^{v}$ of the field $X_{m n}$ is the subspace $H_{X}\left(S^{v}\right)$ and $H_{X}\left(\frac{X}{S^{v}}\right)$, respectively; where $S^{v}=\{(m, n): m \leq 0, n \in Z\}$. (Here and in what follows by $\bar{S}$ we denote the complement of a set $S \subset Z^{2}$; i.e. $\bar{S}=Z^{2} \backslash S$.)

As a measure of the angle between the vertical past-present and future subspaces of the field $X_{m n}$ we take its vertical-cosine defined by

$$
\varrho_{X}^{v}=\sup \left\{|(Y, Z)|: Y \in P_{X}^{v}, Z \in F_{X}^{v},\|Y\|=\|Z\|=1\right\} ;
$$

and the subspaces $P_{X}^{v}$ and $F_{X}^{v}$ are said to be at positive angle if $\varrho_{X}^{v}<1$.

The horizontal past-present subspace $P_{X}^{h}$, the horizontal future subspace $F_{X}^{h}$ and the horizontal-cosine of the angle between these subspaces $\varrho_{X}^{h}$ are defined similarly. Finally we define

$$
\varrho_{X}=\max \left(\varrho_{X}^{v}, \varrho_{X}^{h}\right)
$$

For any nonnegative measure on the torus $\varrho_{\mu}^{v}, \varrho_{\mu}^{h}$ and $\varrho_{\mu}$ can be defined in a similar way. However, if $\mu$ is the spectral measure of our stationary random field $X_{m n}$, then by the Kolmogorov isomorphism it is evident that $\varrho_{X}^{v}=\varrho_{\mu}^{v}, \varrho_{X}^{h}=\varrho_{\mu}^{h}$, and $\varrho_{X}=\varrho_{\mu}$. 
3. Characterizations for the positivity of the angle. In this section we present some geometric and analytic properties of stationary random fields $X_{m n}$ with $\varrho_{X}<1$. This will include the generalizations of some well known geometric and analytic characterizations for the stationary random processes. Our Theorem 3.4 which shows that $\varrho_{X}<1$ if and only if $X_{m n}$ forms a Schauder basis for its time domain seems to be new even in the case of random processes.

The proof of the following lemma is similar to the corresponding result in the case of stationary processes (cf. Helson and Szegö [4]) and is therefore omitted.

3.1. Lemma. Let $X_{m n}$ be a stationary random field, then

(a) $\varrho_{X}^{v}<1$ if and only if there exists a constant $N$ such that

$$
\left\|\sum_{(m, n) \in S^{v}} a_{m n} X_{m n}\right\|_{H} \leq N\left\|\sum_{(m, n) \in Z^{2}} a_{m n} X_{m n}\right\|_{H}
$$

where $\left\{a_{m n}\right\}$ is any double sequence of scalars with finitely many non-zero elements.

(b) $\varrho_{X}^{h}<1$ if and only if there exists a constant $M$ such that

$$
\left\|\sum_{(m, n) \in S^{h}} a_{m n} X_{m n}\right\|_{H} \leq M\left\|\sum_{(m, n) \in Z^{2}} a_{m n} X_{m n}\right\|_{H}
$$

where $\left\{a_{m n}\right\}$ is as in (a).

Now we can prove the following:

3.2. Lemma. Let $X_{m n}$ be a stationary random field. Then $\varrho_{X}<1$ if and only if there exists a constant $K$ such that for any double sequence as in Lemma 3.1(a) and any integers $m_{0}, m_{1}, n_{0}$, and $n_{1}$ we have

$$
\left\|\sum_{m=m_{0}}^{m_{1}} \sum_{n=n_{0}}^{n_{1}} a_{m n} X_{m n}\right\|_{H} \leq K\left\|\sum_{(m, n) \in Z^{2}} a_{m n} X_{m n}\right\|_{H} .
$$

Proof. Using Lemma 3.1(a) and considering the fact that our field is stationary we have

$$
\begin{aligned}
\left\|\sum_{m=m_{0}}^{m_{1}} \sum_{n=-\infty}^{\infty} a_{m n} X_{m n}\right\|_{H} & \leq N\left\|\sum_{m=-\infty}^{m_{1}} \sum_{n=-\infty}^{\infty} a_{m n} X_{m n}\right\|_{H} \\
& \leq N^{2}\left\|\sum_{(m, n) \in Z^{2}} a_{m n} X_{m n}\right\|_{H} .
\end{aligned}
$$

Applying Lemma 3.1(b) in a similar fashion, we get

$$
\left\|\sum_{m=m_{0}}^{m_{1}} \sum_{n=n_{0}}^{n_{1}} a_{m n} X_{m n}\right\|_{H} \leq N^{2} M^{2}\left\|\sum_{(m, n) \in Z^{2}} a_{m n} X_{m n}\right\|_{H} .
$$


To prove the converse take any double sequence $\left\{a_{m n}\right\}$ with finitely many nonzero elements. We know (3.3) is valid for this sequence $\left\{a_{m n}\right\}$. Now taking $m_{1}=0$ and $m_{0}, n_{0}, n_{1}$ large enough one can write (3.3) as

$$
\left\|\sum_{(m, n) \in S^{v}} a_{m n} X_{m n}\right\|_{H} \leq K\left\|\sum_{(m, n) \in Z^{2}} a_{m n} X_{m n}\right\|_{H}
$$

which by Lemma 3.1 implies $\varrho_{X}^{v}<1$. Similarly one can get $\varrho_{X}^{h}<1$ and hence $\varrho_{X}<1$.

Next we show the following useful theorem which states that for a stationary random field $X_{m n}$ the property $\varrho_{X}<1$ is equivalent to the fact that $X_{m n}$ is a Schauder basis for $H_{X}$. Recall that a double sequence $c_{m n}$ is called a Schauder basis for a Hilbert space $H$ if for any element $Z$ in $H$ there exists a uniquely determined set of coefficients $c_{m n}(Z)$ such that

$$
Z=\sum_{m=-\infty}^{\infty} \sum_{n=-\infty}^{\infty} c_{m n}(Z) c_{m n}
$$

We should mention here that by the convergence of a double series

$$
\sum_{m=-\infty}^{\infty} \sum_{n=-\infty}^{\infty} a_{m n}
$$

to a limit we mean that the double sequence of its partial sums converges to that limit and by the partial sums we mean the rectangular partial sums, defined by

$$
S_{m_{0} n_{0}}^{m_{1} n_{1}}=\sum_{m=m_{0}}^{m_{1}} \sum_{n=n_{0}}^{n_{1}} a_{m n} .
$$

Having Lemma 3.2 proved the proof of the following theorem is a standard Schauder basis argument and, in particular, can be given similar to the proof given on pages 102 and 103 of [6], and hence it is omitted.

3.4. Theorem. A stationary random field $X_{m n}$ is a Schauder basis for $H_{X}$ if and only if $\varrho_{X}<1$.

The following lemma shows that the notion of the positivity of angle is stronger than some other measures of independence. The lemma is also useful in our proof of the analytic characterization given in Theorem 3.6. 
3.5. Lemma. Let $X_{m n}$ be a stationary random field. If $\varrho_{X}<1$, then

(a) $X_{m n}$ is horizontally nondeterministic, i.e. $\cap_{p \in Z} H_{X}(\{(m, n): m \leq p$, $n \in Z\})=\{0\}$,

(b) $X_{m n}$ is vertically nondeterministic, i.e. $\cap_{q \in Z} H_{X}(\{(m, n): m \in Z, n \leq q\})$ $=\{0\}$,

(c) $X_{m n}$ is strongly nondeterministic, i.e. $\cap_{p, q \in Z} H_{X}(\{(m, n): m \leq p, n \leq q\})$ $=\{0\}$,

(d) for any two subsets $A$ and $B$ of $Z^{2}$ we have $H_{X}(A \cap B)=H_{X}(A) \cap H_{X}(B)$.

Proof. Since proof of (b) and (c) is similar to (a) we just prove (a) and (d). For the proof of (a) suppose that

$$
Y \in \bigcap_{p \in Z} H_{X}(\{(m, n): m \leq p, n \in Z\}) .
$$

Then $Y \in H_{X}(\{(m, n): m \leq p, n \in Z\})$ for all $p \in Z$. It follows from Theorem 3.4 that $X_{m n}$ is a Schauder basis for $H_{X}$. Hence $\left\{X_{m n}, m \leq p, n \in Z\right\}$ is Schauder basis for $H_{X}(\{(m, n): m \leq p, n \in Z\})$ for each $p$ (cf. the characterization in [6; p. 103]). Thus for each $p \in Z$ we have a representation of the form

$$
Y=\sum_{m=-\infty}^{p} \sum_{n=-\infty}^{\infty} b_{m n}^{p} X_{m n}
$$

But since these representations for $Y$ must be unique, we conclude that $b_{m n}^{p}=b_{m n}^{q}$ for all $p, q, m$ and $n$, yielding $b_{m n}^{p}=0$. Thus, $Y=0$, which completes the proof of (a).

To see (d) take any $Y$ in $H_{X}(A) \cap H_{X}(B)$. Then $Y \in H_{X}(A)$ and $Y \in$ $H_{X}(B)$ so we have

$$
Y=\sum_{(m, n) \in A} a_{m n}^{A} X_{m n} \quad \text { and } \quad Y=\sum_{(m, n) \in B} a_{m n}^{B} X_{m n},
$$

since the elements $X_{m n},(m, n) \in A$, and $X_{m n},(m, n) \in B$, form a Schauder basis in the spaces $H_{X}(A)$ and $H_{X}(B)$, respectively (cf. [6; p. 102]). Since the sequence $X_{m n},(m, n) \in Z^{2}$, is a Schauder basis in $H_{X}$ (cf. Theorem 3.4), it follows from the uniqueness property of the Schauder basis that

$$
a_{m n}^{A}=a_{m n}^{B} \quad \text { for all }(m, n) \in Z^{2} .
$$

Especially

$$
a_{m n}^{A}=a_{m n}^{B}=0 \quad \text { for all }(m, n) \notin A \cap B
$$

yielding

$$
Y=\sum_{(m, n) \in A \cap B} a_{m n}^{A} X_{m n}
$$


Thus, $Y \in H_{X}(A \cap B)$. This shows that $H_{X}(A) \cap H_{X}(B) \subseteq H_{X}(A \cap B)$, and the other inclusion is obviously always correct.

We now prove the following generalization of a well-known analytic characterization for the positivity of the angle between past and future for stationary random processes due to Helson and Szegö [4] and Hunt, Muckenhoupt and Wheeden [5]. (For the matricial form of this result one can see Pousson [11], Pourahmadi [10] and Miamee [8].)

3.6. Theorem. Let $X_{m n}$ be a stationary random field with a spectral measure $\mu$ on the torus. Then, $\mu_{X}<1$ if and only if

(a) $\mu$ is absolutely continuous with respect to the normalized Lebesgue measure $d \sigma=d \alpha d \beta / 4 \pi^{2}$, with spectral density $w$,

(b) $L_{w}^{2} \subset L^{1}$, where $L_{w}^{2}=L_{w d \sigma}^{2}=L_{\mu}^{2}$,

(c) the Fourier series of any $f \in L_{w}^{2}$ converges to $f$ in the norm of $L_{w}^{2}$.

Proof. By Lemma 3.5(c) $X_{m n}$ is strongly nondeterministic in the sense of Soltani [14] and hence by Theorem 3.4 in [14] $\mu$ is absolutely continuous with respect to the Lebesgue measure and its spectral density $w$ has the log property, i.e. $\log w \in L^{1}$. This shows (a).

To see (b) let $I$ be an operator defined on the polynomials

$$
P=\sum_{(m, n) \in Z^{2}} a_{m n} e^{i(m \alpha+n \beta)}
$$

by

$$
I(P)=\iint a_{00} w(\alpha, \beta) d \sigma .
$$

The operator is bounded because

$$
\begin{aligned}
|I(P)| & =\left|\iint a_{00} w(\alpha, \beta) d \sigma\right|=\left|\iint a_{00} \sqrt{w} \sqrt{w} d \sigma\right| \\
& \leq\left(\iint\left|a_{00}\right|^{2} w d \sigma\right)^{1 / 2}\left(\iint w d \sigma\right)^{1 / 2} .
\end{aligned}
$$

Hence by Lemma 3.2 and the Kolmogorov isomorphism we get

$$
|I(P)| \leq K\|P\|_{L_{w}^{2}} \sqrt{\iint w d \sigma .}
$$

Thus $I$ can be extended to a bounded functional on $L_{w}^{2}$. Hence there exists a function $g$ in $L_{w}^{2}$ such that

$$
I(f)=(f, g)_{L_{w}^{2}} \quad \text { for all } f \in L_{w}^{2} .
$$


In particular we have $I\left(e^{i(m \alpha+n \beta)}\right)=\left(e^{i(m \alpha+n \beta)}, g\right)_{L_{w}^{2}}$. On the other hand

$$
I\left(e^{i(m \alpha+n \beta)}\right)= \begin{cases}0, & \text { if }(m, n) \neq(0,0) \\ 1, & \text { if }(m, n)=(0,0)\end{cases}
$$

Thus we have

$$
\iint e^{i(m \alpha+n \beta)} w(\alpha, \beta) \overline{g(\alpha, \beta)} d \sigma= \begin{cases}0, & \text { if }(m, n) \neq(0,0) \\ 1, & \text { if }(m, n)=(0,0)\end{cases}
$$

which means $w \bar{g} \equiv 1$. Hence $w^{-1}=\bar{g} \in L_{w}^{2}$; implying $L_{w}^{2} \subset L^{1}$ because for any $h \in L_{w}^{2}$,

$$
\iint|h| d \sigma=\iint|h| \sqrt{w} \sqrt{w^{-1}} d \sigma \leq\left(\iint|h|^{2} w d \sigma\right)^{1 / 2}\left(\iint w^{-1} d \sigma\right)^{1 / 2} .
$$

To prove (c), by Lemma 3.2 the operators $S_{m n}$ defined on the polynomials $\sum_{(m, n) \in Z^{2}} a_{m n} e^{i(m \alpha+n \beta)}$ by

$$
S_{m n}\left(\sum_{(p, q) \in Z^{2}} a_{p q} e^{i(p \alpha+q \beta)}\right)=\sum_{p=-m}^{m} \sum_{q=-n}^{n} a_{p q} e^{i(p \alpha+q \beta)}
$$

are bounded, with common bound $K$. Hence each of the operators $S_{m n}$ can be extended to a bounded operator on $L_{w}^{2}$, so that they have the same norm $K$. It can be seen that these operators are just the symmetric Fourier partial sum operators. Letting $f \in L_{w}^{2}$, to complete the proof of this part we just have to show that

$$
S_{m n}(f) \rightarrow f, \text { in the } L_{w}^{2} \text { sense. }
$$

Given $\varepsilon>0$, we take a polynomial $P$ such that $\|f-P\|_{L_{w}^{2}}<\varepsilon /(K+1)$, we then have

$$
\begin{aligned}
\left\|S_{m n}(f)-f\right\|_{L_{w}^{2}} & \leq\left\|\left(S_{m n}-I\right)(f-P)\right\|_{L_{w}^{2}}+\left\|S_{m n}(P)-P\right\|_{L_{w}^{2}} \\
& \leq\left\|S_{m n}-I\right\|\|f-P\|_{L_{w}^{2}}+\left\|S_{m n}(P)-P\right\|_{L_{w}^{2}} .
\end{aligned}
$$

Thus

$$
\left\|S_{m n}(f)-f\right\|_{L_{w}^{2}} \leq(K+1) \frac{\varepsilon}{K+1}+\left\|S_{m n}(P)-P\right\|_{L_{w}^{2}} .
$$

Now if we take $m$ and $n$ large enough we get $S_{m n}(P)=P$, and hence

$$
\left\|S_{m n}(f)-f\right\|_{L_{w}^{2}}<\varepsilon .
$$


Now, to prove the other half assume that the conditions (a), (b), and (c) in the statement of the theorem hold. Then by (b) any function $f$ in $L_{w}^{2}$ belongs to $L^{1}$ and, as such, has a Fourier series

$$
f \sim \sum_{(p, q) \in Z^{2}} a_{p q} e^{i(p \alpha+q \beta)} .
$$

We consider the partial sum operator $S_{m_{0} n_{0}}^{m_{1} n_{1}}: L_{w}^{2} \rightarrow L_{w}^{2}$ defined by

$$
S_{m_{0} n_{0}}^{m_{1} n_{1}}(f)=\sum_{p=-m_{0}}^{m_{1}} \sum_{q=-n_{0}}^{n_{1}} a_{p q} e^{i(p \alpha+q \beta)}
$$

For any $f \in L_{w}^{2}$ we can write

$$
\left\|S_{m_{0} n_{0}}^{m_{1} n_{1}}(f)\right\|_{L_{w}^{2}}=\left\|\sum_{p=-m_{0}}^{m_{1}} \sum_{q=-n_{0}}^{n_{1}} a_{p q} e^{i(p \alpha+q \beta)}\right\|_{L_{w}^{2}} \leq \sum_{p=-m_{0}}^{m_{1}} \sum_{q=-n_{0}}^{n_{1}}\left\|a_{p q}\right\|_{L_{w}^{2}} .
$$

Hence

$$
\begin{aligned}
\left\|S_{m_{0} n_{0}}^{m_{1} n_{1}}(f)\right\|_{L_{w}^{2}} & =\sqrt{\iint w d \sigma} \sum_{p=-m_{0}}^{m_{1}} \sum_{q=-n_{0}}^{n_{1}}\left|a_{p q}\right| \\
& \leq\left(2 m_{0} m_{1}+2 n_{0} n_{1}+1\right) \sqrt{\iint w d \sigma}\|f\|_{L^{1}} .
\end{aligned}
$$

Now since $L_{w}^{2} \subset L^{1}$, Lemma 3.1 of Miamee [8] implies that there exists a constant $K$ such that $\|f\|_{L^{1}} \leq K\|f\|_{L_{w}^{2}}$. This means that all operators $S_{m_{0} n_{0}}^{m_{1} n_{1}}$ are bounded. On the other hand, by (c) for each $f \in L_{w}^{2}$ we have

$$
S_{m_{0} n_{0}}^{m_{1} n_{1}}(f) \rightarrow f, \text { in } L_{w}^{2} \text {. }
$$

Hence by the uniform boundedness principle there exists a constant $M$ such that

$$
\left\|S_{m_{0} n_{0}}^{m_{1} n_{1}}\right\| \leq M, \quad \text { for all } m_{0}, m_{1}, n_{0}, n_{1} \geq 0
$$

This means that for any $f \in L_{w}^{2}$ we have

$$
\left\|S_{m_{0} n_{0}}^{m_{1} n_{1}}(f)\right\|_{L_{w}^{2}} \leq M\|f\|_{L_{w}^{2}}, \quad \text { for all } m_{0}, m_{1}, n_{0}, n_{1} \geq 0 .
$$

In particular for any polynomial $P$ we have

$$
\left\|S_{m_{0} n_{0}}^{m_{1} n_{1}}(P)\right\|_{L_{w}^{2}} \leq M\|P\|_{L_{w}^{2}}, \quad \text { for all } m_{0}, m_{1}, n_{0}, n_{1} \geq 0 .
$$

But this, up to the Kolmogorov isomorphism, is just (3.3). Hence by Lemma 3.2 we deduce $\varrho_{X}<1$. 
3.7. Remark. The proof of Theorem 3.6 shows that $w^{-1} \in L^{1}$ and this in turn means that (cf. for example Salehi and Scheidt [12]) the random field $X_{m n}$ with spectral measure $d \mu=w d \sigma$ is minimal, i.e.

$$
X_{m n} \notin H_{X}(\{(p, q):(p, q) \neq(m, n)\}) .
$$

This in particular implies that $X_{m n}$ is purely nondeterministic in the HelsonLowdenslager sense [2]. Hence we have $\iint \log w d \sigma>-\infty$. This also shows that the positivity of angle is stronger than some other measures of independence for a random field.

The following lemma shows that in sharp contrast to other prediction problems for random fields the positivity of the angle is independent of the kind of past one may take, whether it is taken to be the usual half plane [1], the HelsonLowdenslager's half plane [2], [3], or the quarter plane.

3.8. Lemma. If $X_{m n}$ is a stationary random field, then $\varrho_{X}<1$ if and only if the angle between $H_{X}\left(U^{v}\right)$ and $H_{X}\left(\overline{U^{v}}\right)$ as well as the angle between $H_{X}\left(U^{h}\right)$ and $H_{X}\left(\overline{U^{h}}\right)$ are positive, where

$$
U^{v}=\{(m, n): m \leq-1, n \in Z\} \cup\{(0, n): n \leq-1\}
$$

and

$$
U^{h}=\{(m, n): m \in Z, n \leq-1\} \cup\{(m, 0): m \leq-1\} .
$$

If this is the case then the angle between $H_{X}(Q)$ and $H_{X}(\bar{Q})$ is also positive, where $Q$ is the third quadrant, namely

$$
Q=\{(m, n): m \leq 0, n \leq 0\}
$$

Proof. We break the proof of our lemma into the following steps:

Step 1. The angle between $H_{X}\left(U^{v}\right)$ and $H_{X}\left(\overline{U^{v}}\right)$ is positive if and only if there exists a constant $N$ such that

$$
\left\|\sum_{(m, n) \in U^{v}} a_{m n} X_{m n}\right\|_{H} \leq N\left\|\sum_{(m, n) \in Z^{2}} a_{m n} X_{m n}\right\|_{H}
$$

where $\left\{a_{m n}\right\}$ is any double sequence of scalars with finitely many non-vanishing elements. This statement can be proved similar to Lemma 3.1.

Step 2. The angle between $H_{X}\left(U^{v}\right)$ and $H_{X}\left(\overline{U^{v}}\right)$ as well as the angle between $H_{X}\left(U^{h}\right)$ and $H_{X}\left(\overline{U^{h}}\right)$ is positive if and only if there exists a constant $L$ such that

$$
\left\|\sum_{(m, n) \in R} a_{m n} X_{m n}\right\|_{H} \leq L\left\|\sum_{(m, n) \in Z^{2}} a_{m n} X_{m n}\right\|_{H},
$$


where $\left\{a_{m n}\right\}$ is any double sequence of scalars with finitely many non-vanishing elements, and the first summation ranges over any generalized rectangle of the form

$$
R=U_{m_{0} n_{0}}^{v} \cap \bar{U}_{m_{1} n_{1}}^{v} \cap U_{p_{0} q_{0}}^{h} \cap \bar{U}_{p_{1} q_{1}}^{h}
$$

where

$$
U_{m, n}^{v}=\{(r, s): r \leq m-1, s \in Z\} \cup\{(m, s): s \leq n-1\}
$$

and

$$
U_{m, n}^{h}=\{(r, s): r \in Z, s \leq n-1\} \cup\{(r, n): r \leq m-1\} .
$$

The proof of this step is similar to that of Lemma 3.2 and hence it is again omitted.

Step 3. We note that the generalized rectangles contain all the usual rectangles. Thus to complete the proof of the lemma it suffices to show that $\varrho_{X}<1$ implies (3.9). To see this we observe that any region $R$ in (3.9), that is any region $R$ of the form (3.10), can be represented as a disjoint union of at most 5 usual rectangles, say $R_{i}, i=1,2,3,4,5$. Thus we can take the $L$ in Step 2 to be simply $5 M$. In fact, for any $R$ of the form in (3.9) or (3.10) we can write

$$
\begin{aligned}
\left\|\sum_{(m, n) \in R} a_{m n} X_{m n}\right\|_{H} & \leq \sum_{i=1}^{5}\left\|\sum_{(m, n) \in R_{i}} a_{m n} X_{m n}\right\|_{H} \\
& \leq \sum_{i=1}^{5} M\left\|\sum_{(m, n) \in Z^{2}} a_{m n} X_{m n}\right\|_{H} \\
& \leq 5 M\left\|\sum_{(m, n) \in Z^{2}} a_{m n} X_{m n}\right\|_{H}
\end{aligned}
$$

\section{References}

[1] ChiAng, TsE-PEI: On the linear extrapolation of a continuous homogeneous random field. - Theor. Probab. Appl. 2, 1957, 58-88 (English translation of Teor. Veroyatnost. i Primenen. 2, 1957, 60-91).

[2] Helson, H., and D. Lowdenslager: Prediction theory and Fourier series in several variables, I. - Acta Math. 99, 1958, 165-202.

[3] Helson, H., and D. Lowdenslager: Prediction theory and Fourier series in several variables, II. - Acta Math. 106, 1959, 175-213.

[4] Helson, H., and G. Szegö: A problem in prediction theory. - Ann. Mat. Pura. Appl. (4) $51,1960,107-138$.

[5] Hunt, R., B. Muckenhoupt, and R.L. Wheeden: Weighted norm inequalities for the conjugate function and Hilbert transform. - Trans. Amer. Math. Soc. 176, 1973, 227-251. 
[6] LACEY, H.E.: The isometric theory of classical Banach spaces. - Springer-Verlag, BerlinHeidelberg-New York, 1974.

[7] Makagon, A., and H. Salehi: Stationary fields with positive angle. - J. Multivariate Anal. 22, 1987, 106-125.

[8] Miamee, A.G.: On the angle between past and future and prediction theory of stationary stochastic processes. - J. Multivariate Anal. 20, 1986, 205-219.

[9] Miamee, A.G., and H. Niemi: On the angle for stationary random fields. - Tech. Report 92, Center for Stochastic Processes, University of North Carolina, Chapel Hill, 1985.

[10] Pourahmadi, M.: A matrical extension of the Helson-Szegő theorem and its application in multivariate prediction. - J. Multivariate Anal. 16, 1985, 265-275.

[11] Pousson, H.R.: Systems of Toeplitz operators on $H^{2}$, II. - Trans. Amer. Math. Soc. 133, $1968,527-536$.

[12] SALEHI, H., and J.K. SCHEIDT: Interpolation of $q$-variate stationary stochastic processes over locally compact abelian group. - J. Multivariate Anal. 2, 1972, 307-331.

[13] Sarason, D.E.: Function theory on the unit circle. - Lecture Notes, Polytechnic Institute and State University, Blackburg, Virginia, 1978.

[14] Soltani, A.R.: Extrapolation and moving average representation for stationary random fields and Beurling's theorem. - Ann. Probab. 12, 1984, 120-132. 